\title{
REPRESENTACIONES MÚLTIPLES Y COMPRENSIÓN DE DOCUMENTOS EN EL DOMINIO DE LA TECNOLOGÍA ${ }^{1}$
}

\author{
Luis Facundo Maldonado Granados, Ph.D. \\ Juan Bautista Se queda Tarazona.
}

\begin{abstract}
Human knowledge is mainly found in printed documents. Among them, technical documents compound a relevant type of literature whose understanding a is an effort consuming task This article is dealing with an experimental study which compares three representation strategies: the fist one is only text based, the second uses graphics and diagrams together with text and the last one is simulation addressed. A sample of 85 tenth grade students from a technical oriented school was randomly assigned to each of the experimental conditions. A pretest - posttest experimental design was conducted with an additional retention test. The results show that diagrams and graphics ease text comprehension, and simulation increases retention rate.
\end{abstract}

\section{RESUMEN}

La mayor parte del conocimiento humano se conserva en documentos escritos. Entre estos ocupan un lugar importante los textos sobre temas técnicos. Su comprensión es una tarea difícil para la mayoría de los lectores. Este artículo describe un experimento que compara tres formas de representación de un mismo tema técnico usando una plataforma hipertextual: una que utiliza sólo texto, otra que acompaña el texto con diagramas y gráficas y la tercera que utiliza texto y simulaciones. Los sujetos fueron 85 estudiantes de décimo grado de un colegio con orientación técnica, los cuales fueren asignados aleatoriamente a una de las tres condiciones. Se utilizó un diseño experimental pretestpostest y una medición adicional de retención. Los resultados muestran que con diagramas y gráficas el texto es más comprensible, pero, que con animaciones, la tasa de retención es mayor.

Palabras claves: Representaciones múltiples, imaginería mental, animación mental, motores de inducción, motores monofásicos de inducción, hipertextos, ambientes de aprendizaje basados en computador.

\footnotetext{
${ }^{1}$ La investigación a la que hace referencia este artículo fue dirigida por Luis Facundo Maldonado Granados, Ph.D., profesor titular de la Universidad Pedagógica Nacional y director del Grupo TECNICE de investigación, y presentada como tesis para obtener el titulo de Magíster en Tecnologías de la Información Aplicadas a la Educación por Juan Bautista Sequedad Tarazona, M.Sc. Profesor del INEM de Bucaramanga.
} 


\section{INTRODUCCIÓN}

A diario, nos vemos enfrentados a la lectura de mensajes, cualquiera sea su presentación, ya sea en libros, revistas, periódicos, programas de computador, videos, etc.; unos con información muy valiosa, otros menos, pero, ¿comprendemos a cabalidad la información contenida en ellos? ¿Se aprovecha en su totalidad toda la información? Seguramente se obtendrán diferentes respuestas, pero, la verdad es que no todos comprendemos y entendemos al mismo nivel, ni en el mismo grado; no todos poseemos la misma capacidad de asimilación, ni conocemos estrategias para la efectiva comprensión de documentos.

Por lo tanto, interesa encontrar algunas estrategias o medios que conlleven a mejorar y facilitar la comprensión de documentos -específicamente de hipertextos basados en computador-, y para tal fin, se formulan las siguientes preguntas: ¿La introducción de animaciones incrementa la comprensión de los hipertextos?; ¿El incremento de gráficos o esquemas inciden en la mejor comprensión de los hipertextos?

La literatura existente al respecto es bastante variada y se encuentran diferentes teorías y enfoques que estudian el problema de comprender un texto.

\section{La comprensión de textos}

Otero J. (1990) estudia dos problemas en concreto: 1) el papel de los esquemas del que aprende - problemas de naturaleza cognitiva-, y 2) el control de la propia comprensión al procesar un texto científico — problemas de orden metacognitivo-. Kieras (1985, en Otero J. 1990) dice: "La característica principal de la prosa técnica es que, en general, su contenido es básicamente nuevo para el que lee, por ejemplo, un libro de texto $\mathrm{y}$, por tanto, no se pueden aplicar de manera apreciable esquemas de contenido. Esto sugiere que la comprensión de prosa técnica no es fundamentalmente una cuestión de concordancia entre el contenido y un patrón conocido previamente, sino que implica tratar con el contenido del pasaje al nivel de las proposiciones individuales que expresan el contenido" (p. 94).

En el segundo problema, y en relación con los textos científicos, el sujeto puede carecer de esquemas adecuados y ser consciente de que no puede organizar convenientemente las proposiciones del texto, o no serlo y activar, entre los posibles esquemas, uno inadecuado.

Para Kintsch (1994) recordar no es de ninguna manera equivalente a aprender un texto. Recordarlo significa que uno lo puede reproducir más o menos al pie de la letra y más o menos completo. Por otra parte, aprenderlo implica que uno es capaz de usar la información suministrada por el texto de otras maneras, no simplemente para su reproducción.

Normalmente la reproducción de un texto y su comprensión se correlacionan, de manera que memorizar un texto llega a ser un requisito previo para aprenderlo, aunque, no necesariamente. Por ejemplo, Bransford, Barclay y Franks (1972) en Walter kintsch (1994) han mostrado que las personas pueden adquirir un modelo mental útil sin memorizar el texto, aunque otros (p. ej., Moravcsik y kintsch, 1993) han encontrado que, a 
veces, es posible recordar un texto bastante bien sin ser capaz de usar la información productivamente.

Van Dijk y kintsch (1983) y Kintsch (1992) distinguen diferentes niveles de comprensión. Un componente superficial está constituido por las palabras y frases codificadas y las relaciones lingüísticas. La estructuras semántica y retórica proveen otro nivel de relaciones que son importantes en la comprensión y que frecuentemente son codificadas; este nivel se ha llamado el texto base. El modelo situacional, por otra parte, corresponde al nivel más profundo de entendimiento; en éste, la información proveída por el texto es elaborada y los conocimientos previos se integran con él. Estos tres niveles de análisis - la estructura superficial, el texto base y el modelo situacional- son representados como relaciones en una red de proposiciones.

Mannes y Kintsch (1987, en Walter kintsch, 1994) usaron el paradigma del organizador previo. Obtuvieron como resultado, que para memorizar texto, el texto base es muy bueno, pero no para hacer inferencias y solucionar problemas, los cuales dependen más del modelo situacional.

Por otra parte, el contenido, la forma y el tipo de usuarios a quienes va dirigido son aspectos importantes en la comprensión de un texto. Está bien establecido que los conocimientos generales son importantes para la memorización y comprensión. A veces, los lectores comprenden exitosamente documentos sobre temas a cerca de los cuales no tienen conocimientos generales o específicos, pero necesitan ayudarse de un estilo literario que les permita aplicar estrategias generales de comprensión para sustituir los conocimientos de los cuales carecen (Moravcsik y Kintsch, 1993).

Una escritura buena y uniforme despeja las dificultades para los lectores y hace la comprensión fácil, minimizando lo que lectores necesitan hacer por sí mismos. Todo está allí, bien organizado y listo para ser asimilado. Sin embargo, ésta no es necesariamente la mejor condición para aprender. Lo que necesitamos son textos que provean a los lectores oportunidades para usar sus conocimientos, esto es, textos que no sean bien escritos en el sentido de que lo dicen todo, sino que dejan espacios para que los lectores llenen. A nivel local puede significar que las elaboraciones seguras no son dadas en el texto, sino que son puestas para que el lector las genere; las relaciones coherentes no se especifican siempre, para que así el lector las pueda inferir; la información necesaria de soporte no se suministra, con la esperanza de que los lectores puedan recordar de su memoria acumulada. A nivel general esto podría significar que la macroestructura del texto no es señalada claramente, sino que es insinuada para que el lector la deduzca.

Vygotsky (1934/1986) en Walter Kintsch (1994) introducen el concepto de zonas próximas de desarrollo: áreas alrededor de las cuales se sabe que el crecimiento futuro o aprendizaje toman lugar exitosamente. El concepto de zona puede también ser útil para comprender el aprendizaje de textos. Cada lector puede ser caracterizado por cierto dominio del conocimiento y ciertas habilidades que sirven de base para el aprendizaje exitoso.

En general, la superposición de contenidos del el texto con los conocimientos del lector parece ser una condición necesaria para aprender del texto. Los textos que son óptimos para aprender superpondrán suficientes contenidos con conocimientos del lector, dejando un conjunto no vacío de contenidos no correspondientes con conocimientos. Los textos fuera de la zona de aprendizaje —-superposición nula - pueden recordarse, pero es muy Digitalizado por RED ACADEMICA 
probable que permanezcan como conocimientos inertes, sin integración con los conocimientos previos. Nosotros no hemos probado aún esta hipótesis, pero las evidencias presentadas la hacen sugestiva.

Yulin Qin y Herbert Simon (1995) concluyen que la lectura y el entendimiento son procesos que generan cambios en los modelos mentales de los sujetos. Estos modelos proveen el substrato para formar imágenes mentales transitorias. Están compuestos por formas de memoria que ayudan a los sujetos a formar las representaciones nuevas que necesitan para resolver problemas.

Narayanan, Suwa, \& Motoda (1995), definen el razonamiento diagramático como "el acto de razonamiento, y solución de problemas, usando diagramas como representaciones externas'. Aunque los diagramas son representaciones externas estáticas, razonando con ellos envuelven simulación mental de los cambios del comportamiento de las configuraciones descritas en ellas, los cuales después pueden ser soportadas por descripción de procesos cognitivos que son imágenes en natural.

\section{La comprensión de diagramas}

Hegarty (1995) define animación mental como el proceso de inferir la cinemática de un sistema mecánico desde una configuración estática del sistema. La investigación sugiere que cuando la gente anima mentalmente, descompone la representación en elementos más pequeños correspondientes a la máquina y anima estos componentes en una secuencia que corresponde a la cadena causal de eventos en la operación de la máquina.

Blackwell (1997) se hace la pregunta: ¿Cómo los diagramas se relacionan con las cosas que suceden dentro de nuestras cabezas? Como respuesta, presenta un compendio de investigaciones empíricas y teóricas relacionadas con estos procesos. Considera los orígenes, la interpretación y a manipulación de los diagramas.

\section{Comparación de tres formas de representación de información técnica}

Como alternativa tendiente a buscar estrategias conducentes a mejorar la lectura y comprensión de textos e integrar los conceptos y teorías expuestas por Kintsch (1994), Qin y Simon (1995), Narayanan y Suwa, \& Motoda (1995), Hegarty (1995), se llevó a cabo un experimento multimedial para la comprensión de un mismo texto base, mediante tres versiones diferentes de software. Una primera presenta el texto acompañado de animaciones de los principios expuestos en él, una segunda versión es totalmente textual y en una tercera presenta el texto con gráficos y esquemas. El dominio de conocimiento fue los bobinados en cadena de motores monofásicos de inducción.

Se parte del supuesto de que las experiencias previas con textos, gráficos y animaciones, pueden, en sus diferentes combinaciones, crear una base diferente de prerrequisitos para la comprensión de mensajes. Los sujetos estarían en condiciones diferentes de comprensión de la información contenida en las tres versiones de software y, por ende, la modificación de sus preconceptos y el cambio en sus modelos mentales, operaría, como consecuencia, también de manera diferente.

Para el experimento se tomó como texto base el documento titulado "Bobinados en cadena de motores monofásicos de inducción" (SINGER, 1976), como variables 
independientes, las tres versiones de software y como variable dependiente la comprensión del texto. Se plantearon las siguientes hipótesis: $\mathrm{H}_{0}$ : no existen diferencias significativas en la comprensión del texto, entre los alumnos de décimo grado que utilizan un software con animación en comparación con quienes usan un software gráfico y los que usan un software hipertextual; $\mathrm{H}$.: el número de respuestas acertadas es mayor para los alumnos que utilizan el software con animación que para los que utilizan el software gráfico o el software hipertextual; y $\mathrm{H}$.. el número de respuestas acertadas es mayor en los alumnos de décimo grado que utilizan el software gráfico que en los alumnos que utilizan el software hipertextual. La metodología empleada se condensa en la gráfica 1.

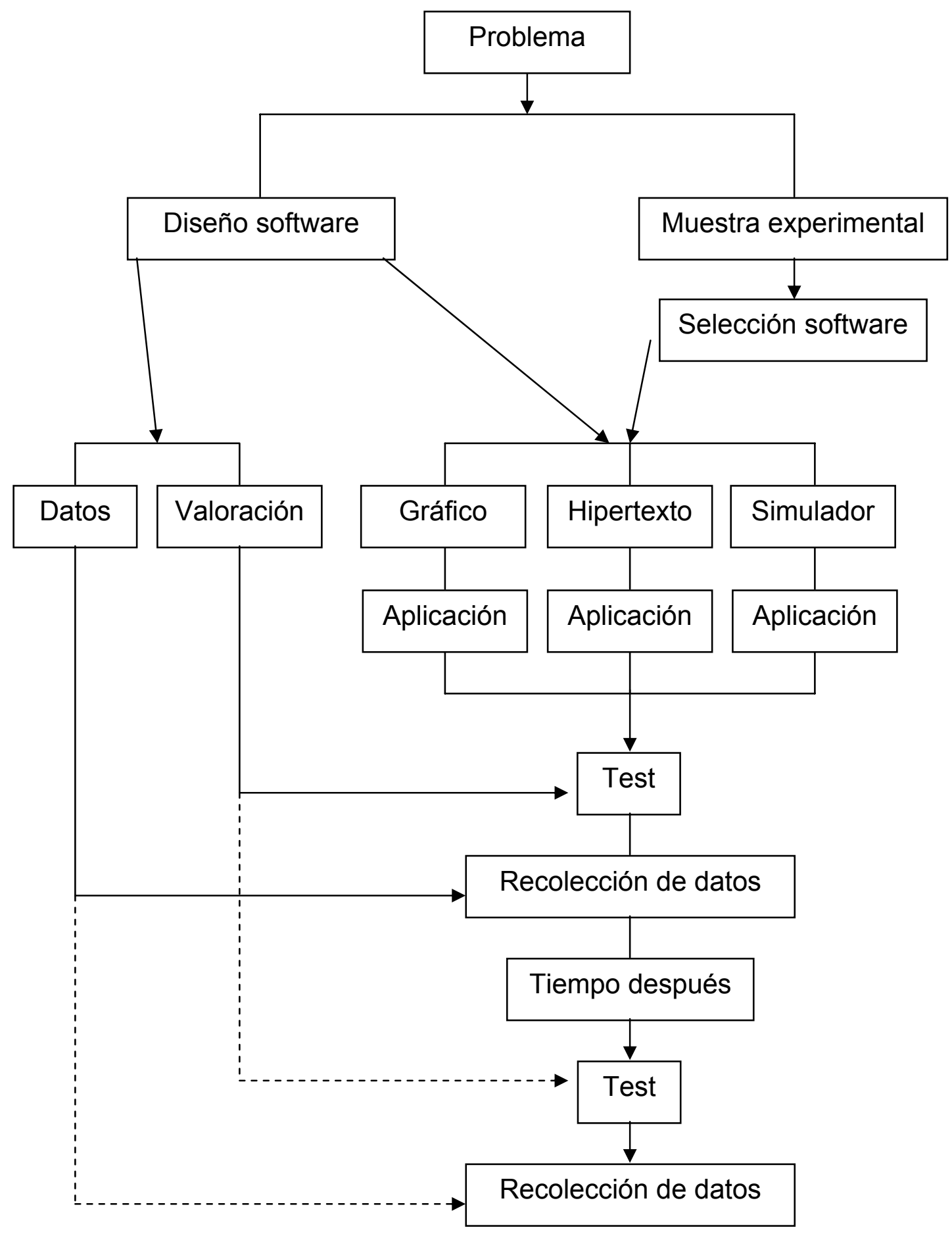

Gráfica 1. Esquema general de la metodología. 
Las tres versiones del software experimental se realizaron en la plataforma Toolbook ${ }^{2}$ versión 6.5; de igual forma, se desarrolló un software para la aplicación del test y recolección de datos.

La gráfica 2 muestra el esquema general de entrada a cualquiera de las versiones del software de aplicación de la investigación. Al iniciar el programa el computador presenta una página con las instrucciones generales, luego el usuario debe registrar su nombre para poder acceder al menú principal que presenta cuatro opciones para navegar.

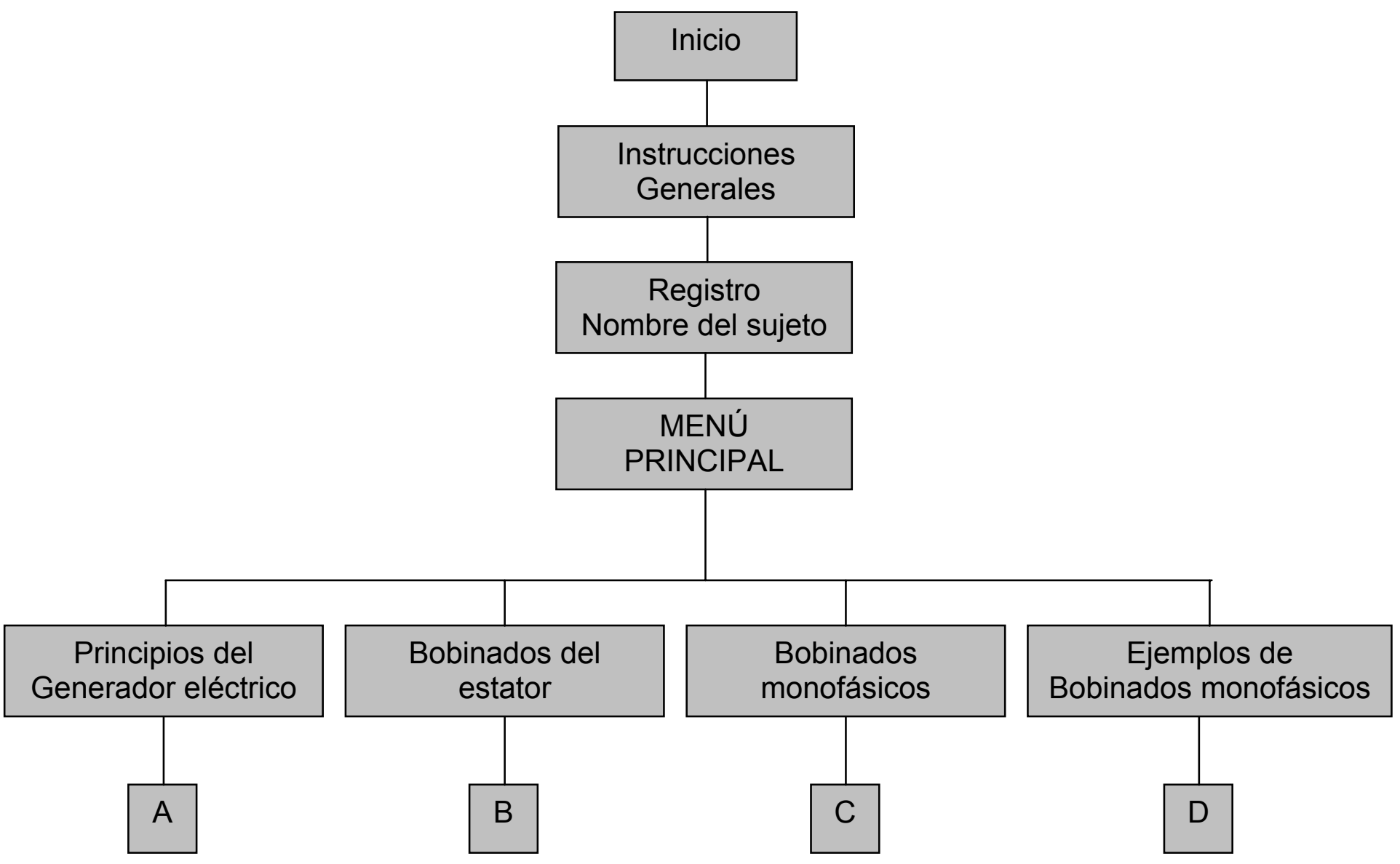

Gráfica 2. Entrada general del software.

La opción A describe dos principios básicos en los cuales se fundamenta el generador eléctrico y algunas reglas prácticas para determinar el sentido del movimiento de un conductor dentro de un campo magnético y la polaridad de la tensión generada en él.

En la opción $B$ se describen dos aspectos fundamentales de los motores monofásicos de inducción, a saber: 1) Factores característicos tales como velocidad, número de polos, número total de ranuras y número de ranuras por polo y por fase, y 2) Tipos de bobinados, entre los que se describen: concéntricos, distribuidos y de barras.

\footnotetext{
${ }^{2}$ ToolBook es marca registrada de Asymetrix Corporation.
} 
La opción C trata los siguientes aspectos: 1) Tipos de motores monofásicos clasificados en motores de fase partida, de condensador y de repulsión, 2) Arrollamientos estatóricos: arrollamiento principal y auxiliar; 3) Conexión entre grupos: alternante y conexión consecuente; 4) Ejemplo de aplicación.

En la opción D se plantean ejercicios para solucionar, dado el número de ranuras y el número polos.

La muestra experimental se tomó de tres secciones del grado décimo de un colegio oficial con orientación técnica - INEM de la ciudad de Bucaramanga-. De cada sección se seleccionaron 32 alumnos al azar mediante fichas de papel, unas con la leyenda Si y otras con la leyenda NO. Los que sacaron la ficha Si participaron en el experimento y los de la ficha No fueron excluidos. Posteriormente, los sujetos de la muestra experimental se asignaron al azar, mediante balotas de colores, a una versión del software.

En el aula de informática, a cada estudiante se le asignó un computador y se organizaron en forma consecutiva según la versión del software. Durante un tiempo de una hora los sujetos exploraron y estudiaron el software correspondiente. Al final de este período se les concedió un descanso de 10 minutos, para luego continuar con la solución de un cuestionario de 40 preguntas seleccionadas del tema estudiado, contando para ello con un tiempo de 30 minutos. Pasada la media hora, o silos estudiantes terminaban antes del tiempo previsto, el computador les mostraba por pantalla un reporte del tiempo empleado, número de aciertos, número de errores y su equivalencia en porcentaje. Al finalizar la sesión los datos quedaron registrados en el disco duro del computador.

A los ocho días, en idénticas condiciones y sin lectura del texto se aplicó la misma prueba, con el fin de evaluar la retención de la información comprendida.

\section{RESULTADOS}

Para facilitar el análisis de datos, los grupos experimentales se identifican de la siguiente forma:

1. Grupo 1. Hipertexto, diagramas y gráficas.

2. Grupo 2. Sólo hipertexto.

3. Grupo 3. El hipertexto con animaciones.

Tabla 1. Medias, diferencia entre Medias y número de casos para cada grupo.

\begin{tabular}{|c|c|c|c|c|}
\hline \multirow{2}{*}{$\begin{array}{c}\text { GENERAL } \\
\text { MANOVA }\end{array}$} & \multicolumn{4}{|c|}{ Rao R $(4,164)=4.05 ; p<0037$} \\
\cline { 2 - 5 } & REND1 & REND2 & DIFEREN & Valid N \\
\hline 1 & 19.519 & 16.704 & 2.815 & 27 \\
\hline 2 & 16.897 & 14.759 & 2.138 & 29 \\
\hline 3 & 16.6 & 15.9 & 0.7 & 30 \\
\hline
\end{tabular}


La tabla 1 muestra las Medias obtenidas por cada grupo en las dos aplicaciones del test —REND1 y REND2_; la diferencia entre ellas —DIFEREN_; y el número de casos válidos para cada grupo — Valid N-. Datos que dan soporte a las gráficas 3 y 4.

En la gráfica 3 sobre el eje $X$ se tabuló la condición experimental, CEXP1, es decir, el número de grupos experimentales. Los puntos G1:1, G2:2 y G_3:3 corresponden al grupo 1, grupo 2 y grupo 3 respectivamente. Sobre el eje $Y$ se tabularon las variables dependientes o Medias de los datos obtenidos después de la aplicación de los tests. La línea azul muestra la curva correspondiente a la variable dependiente REND1, o Medias obtenidas por cada grupo experimental en la primera aplicación del test, y la línea roja de trazos muestra la curva correspondiente a la variable dependiente REND2, o Medias obtenidas por cada grupo experimental en la segunda aplicación del test, transcurridos ocho días de la primera aplicación.

Según los datos de la tabla 1, en la primera aplicación del test, existen diferencias apreciables entre las Medias obtenidas por cada grupo experimental. Entre el grupo 1 y el grupo 2 existe una diferencia de $2.622(19.519-16.897=2.622)$, entre el grupo 1 y el grupo 3 una diferencia de 2.919(19.519-16.6 =2.919), y entre el grupo 2 y el grupo 3 una diferencia de $0.297(16.897-16.6=0.297$.

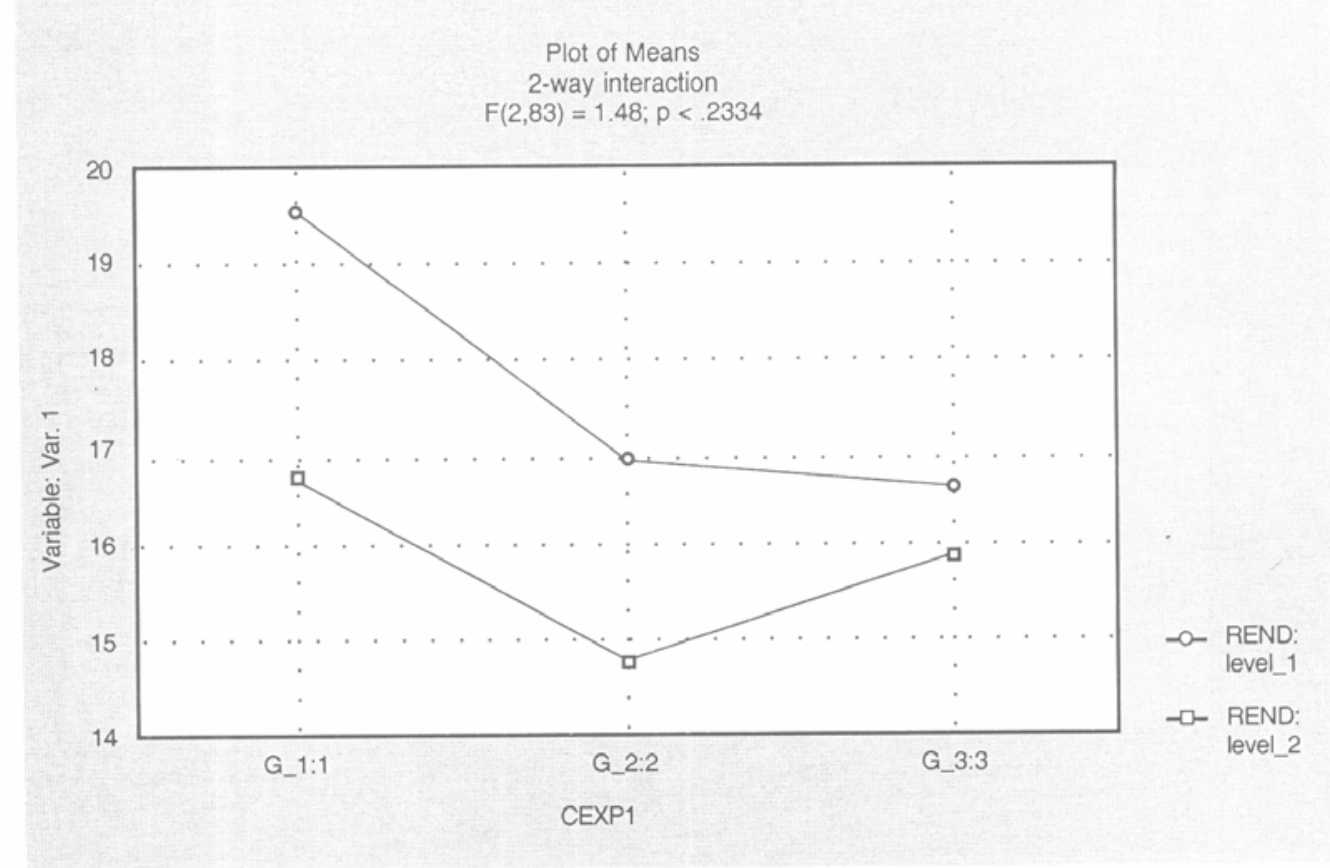

Gráfica 3. Rendimiento obtenido en la primera y segunda aplicaciones del test.

De igual forma, en la segunda aplicación del test, también se presentaron diferencias entre las Medias de cada grupo experimental. La diferencia de Medias entre el grupo 1 y el grupo 2 tiene un valor de $1.945(16.704-14.759=1.945)$, la diferencia de Medias entre el grupo $1 \mathrm{y}$ el grupo 3 es de 0.804 (16.704 - $15.9=0.804)$; y la diferencia de Medias entre el grupo 2 y el grupo 3 es de $-1.141(14.759-15.9=-1.141)$.

La gráfica 4 representa la diferencia de Medias entre la primera y segunda aplicación para los tres grupos experimentales. 
El grupo 1 obtuvo una diferencia de 2.815 entre las dos aplicaciones del test (ver tabla 1) y está representada en la gráfica con el punto GD_1:1; el grupo 2, de 2.138 - punto G_2:2 - finalmente, el grupo 3, 0.700 - punto G_3:3 -.

Hasta aquí los datos muestran dos tipos de diferencias: 1) - de rendimiento entre la primera y segunda aplicación del test, y 2) - entre las Medias de los grupos experimentales. Pero, ¿qué tan significativas son estas diferencias?, Un análisis de varianza ayudará a resolver el interrogante.

La tabla 2 muestra el resumen de los datos obtenidos del análisis de varianza aplicado a la diferencia de rendimiento entre la primera y segunda aplicación. Los resultados muestran que la diferencia como tal no es significativa, pues el nivel de probabilidad de error ( $p$-level) es mayor a 0.05 y el cociente $F$ es pequeño debido a que la varianza dentro de los grupos es relativamente grande (22.6966) comparado con la varianza entre grupos (33.6062).

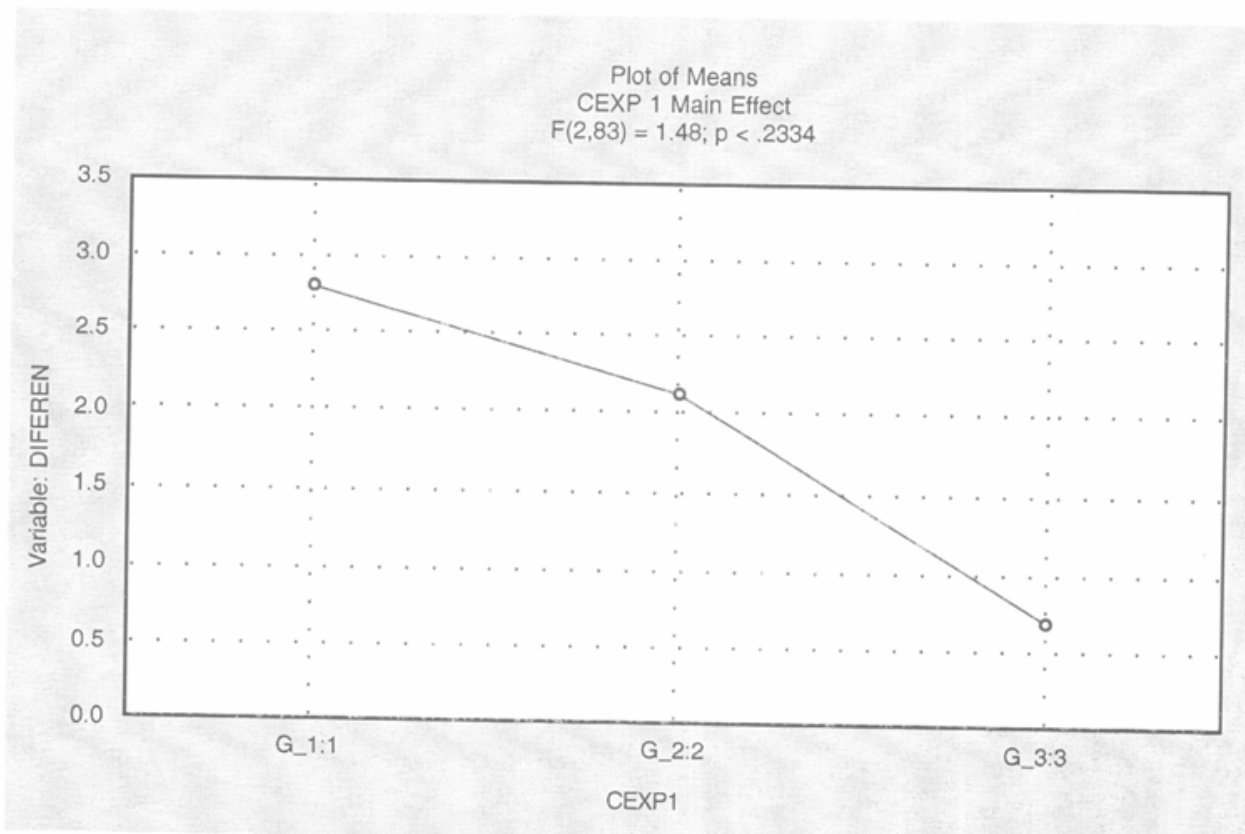

Gráfica 4. Diferencias entre medias de cada grupo experimental.

Tabla 2. Análisis de varianza, diferencia de rendimiento entre las dos aplicaciones del test.

\begin{tabular}{|l|l|l|l|l|l|l|}
\hline \multirow{2}{*}{ Effect } & 1-CEXP1 & & & & & \\
\cline { 2 - 7 } & df Effect & MS Effect & df Error & MS Error & F & p-level \\
\hline 1 & 2 & 33.606266 & 83 & 22.6966553 & 1.48067045 & 0.23343115 \\
\hline
\end{tabular}

La tabla 3 muestra los datos obtenidos en el análisis de varianza aplicado a las diferencias entre las medidas de los grupos experimentales, o análisis de varianza de medidas repetidas. Los resultados son significativos porque la probabilidad de error ( $p$-level) es inferior a 0.05 y el cociente $\mathrm{F}$ es alto. 
Para que el resultado del cociente $\mathrm{E}$ sea significativo se requiere que en CEXP1 para 2 y 83 grados de libertad sea mayor a 3.1, y en el factor REND para 1 y 83 grados de libertad sea mayor a 3.96, por lo tanto, los valores de los cocientes obtenidos 6.676 y 13.427 son altamente significativos.

Los datos de la tabla 4 muestran el promedio de la suma de las Medias obtenidas en las dos aplicaciones del test para cada grupo experimental, CEXP1, y dan soporte a la gráfica 5 .

Tabla 3. Análisis de varianza, diferencia de Medias entre grupos.

\begin{tabular}{|c|c|c|c|c|c|c|}
\hline \multirow{2}{*}{ Effect } & 1-CEXP1 & & & & & \\
\cline { 2 - 7 } & df Effect & MS Effect & df Error & MS Error & F & p-level \\
\hline 1 & 2 & 81.905 & 83 & 12.269 & 6.676 & 0.002 \\
\hline 2 & 1 & 152.373 & 83 & 11.348 & 13.427 & 0.000 \\
\hline 12 & 2 & 16.803 & 83 & 11.348 & 1.481 & 0.233 \\
\hline
\end{tabular}

Tabla 4. Medias promediadas CEXP1.

\begin{tabular}{|c|c|}
\hline \multirow{2}{*}{ CEXP1 REND } & $F(2.83)=6.68 ; p<.0020$ \\
\cline { 2 - 2 } & $\begin{array}{c}\text { Depend } \\
\text { Var. } 1\end{array}$ \\
\hline $1 \ldots .$. & 18.111 \\
\hline $2 \ldots$. & 15.828 \\
\hline $3 \ldots$ & 16.250 \\
\hline
\end{tabular}

Gráfica 5. Promedio de Medias para cada grupo experimental.

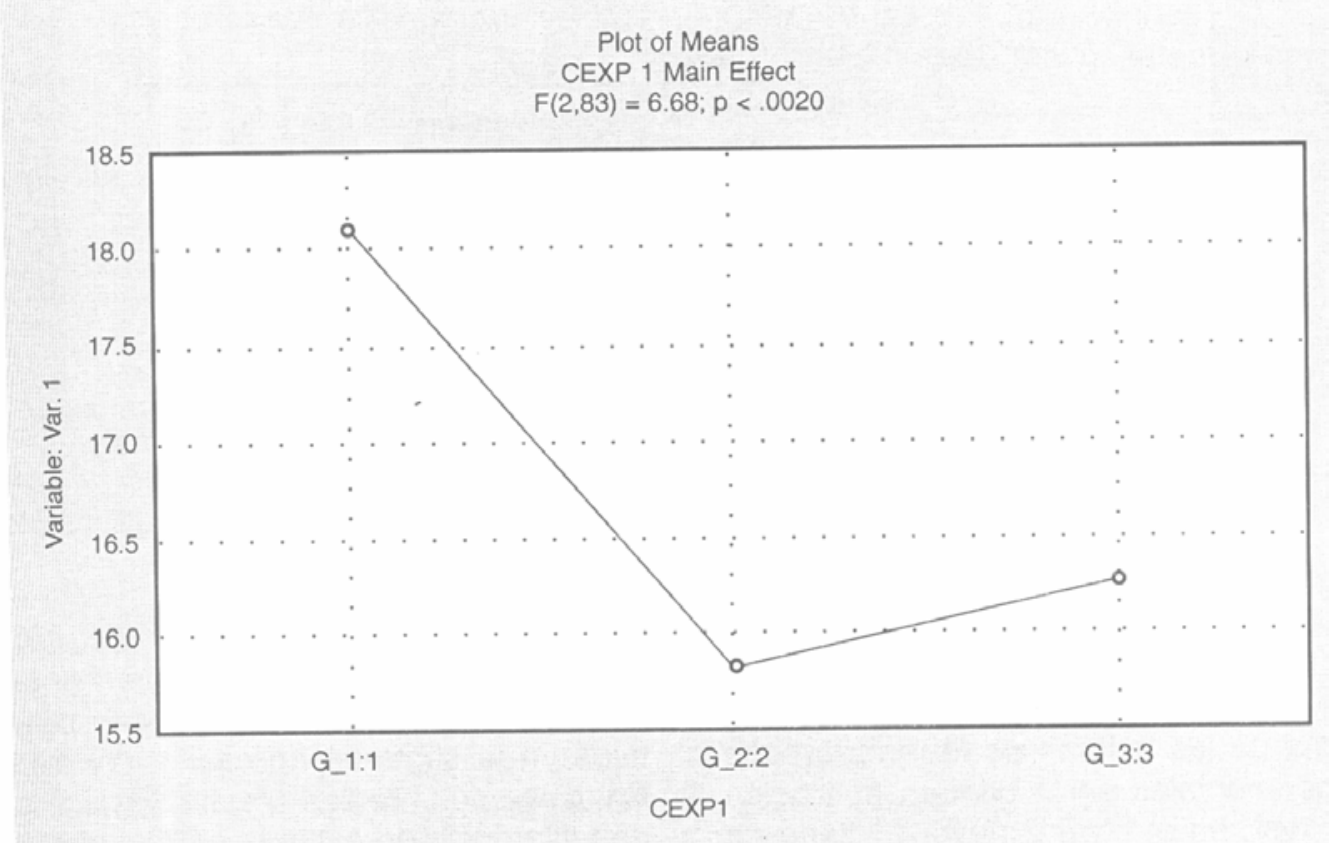


La gráfica 5 Muestra el promedio de las Medias para los tres grupos experimentales. El grupo 1 obtuvo la Media mayor con un valor de 18.111 (ver tabla 4), seguido por el grupo 3 con una Media de 16.250 (ver tabla 4), y el grupo 2 obtuvo la Media menor con un valor 15.828 (ver tabla 4). La diferencia de Medias entre el grupo y el grupo 3 es de $1.861(18.111-16.250=1.861)$, entre el grupo 1 y el grupo 2 es de 2.283 (18.111$15.828=2.283)$, y entre el grupo 3 y el grupo 2 es de $0.422(16.250-15.828=0.422)$.

Los datos de la tabla 5 muestran los resultados del rendimiento total para los tres grupos experimentales, agrupados bajo la variable REND, y dan soporte a la gráfica 6 .

Tabla 5. Medias totales, factor REND.

\begin{tabular}{|cc|c|}
\hline CEXP1 & REND & $\mathrm{F}(1.83)=13.43 ; \mathrm{p}<.0004$ \\
\cline { 3 - 3 } & $\begin{array}{c}\text { Depend } \\
\text { Var. 1 }\end{array}$ \\
\hline$\ldots .1$ & 17.672 & \\
\hline$\ldots 2$ & 15.787 & \\
\hline
\end{tabular}

Gráfica 6. Medias totales, Factor REND.

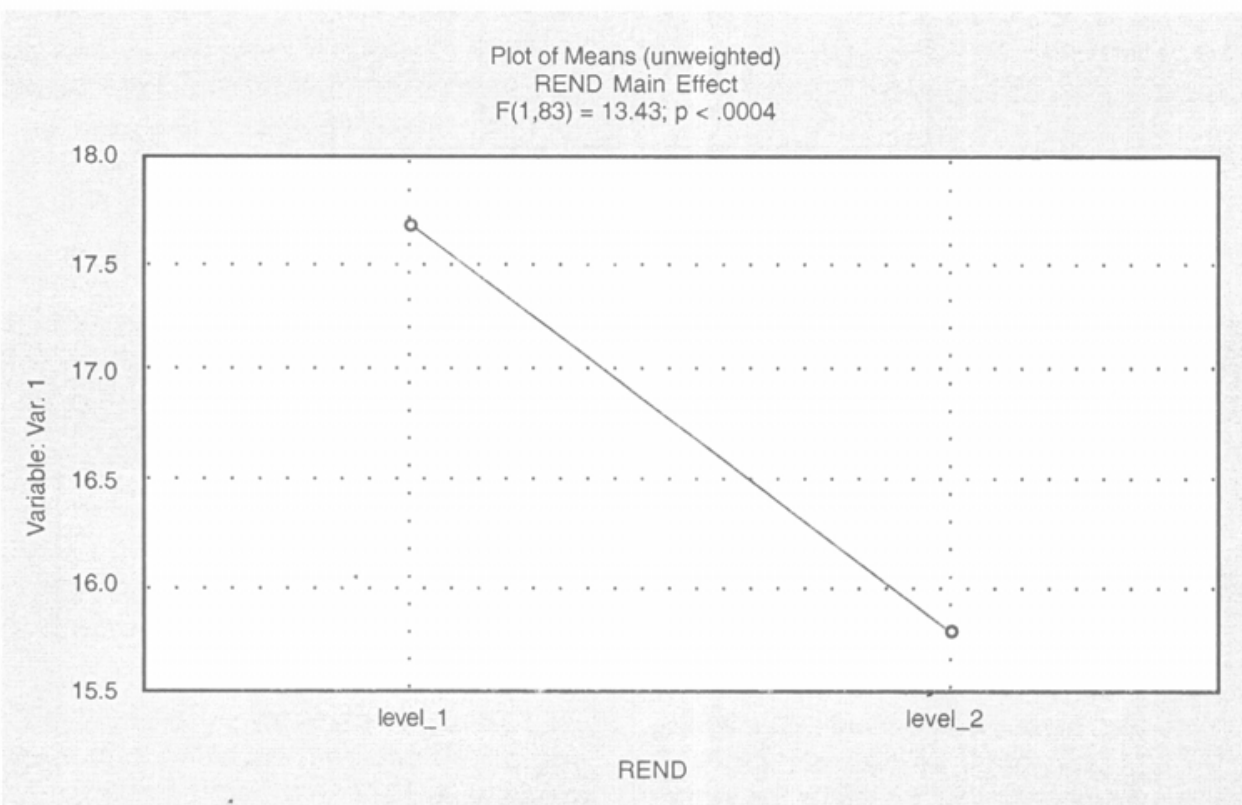

En la gráfica 6, el nivel 1 (level_1) tiene un valor de 17.672 (ver tabla No. 5), dato que corresponde al promedio de la suma de las Medias de los tres grupos experimentales en la primera aplicación del test, en el nivel 2 (level_2) tiene un valor de 15.787 (ver tabla No. 5 ), dato que corresponde al promedio de la suma de las Medias de los tres grupos experimentales en la segunda aplicación del test. Entre las dos Medias resultantes existe una diferencia de $1.885(17.672$ - $15.787=1.885)$, la cual se traduce como pérdida de la información debida al transcurso del tiempo. 
La pérdida de la información por efecto del paso del tiempo es un problema de orden cognitivo donde la memoria a largo plazo juega un papel fundamental. En la memoria episódica la recuperación de los episodios pasados no es completa sino restringida, es decir, detalles del contexto se pierden con el tiempo. En la memoria semántica, la información puede tener deficiencias en su organización y en su articulación a estructuras cognitivas preexistentes, dando lugar a la pérdida de sentido de algunas proposiciones y como consecuencia, a la pérdida de información.

La tabla 6 muestra los datos correspondientes a las Medias obtenidas por cada grupo experimental en forma independiente en las dos aplicaciones del test (Rend 1 y Rend 2), datos utilizados para dar soporte a la gráfica 7.

Tabla 6. Medias obtenidas en la primera y segunda aplicación del test.

\begin{tabular}{|c|c|c|}
\hline & & $\begin{array}{c}F(2.83)=1.48 ; p<.2334 \\
\text { Depend }\end{array}$ \\
\hline CEXP1 & REND & Var.1 \\
\hline 1 & 1 & 19.519 \\
\hline 1 & 2 & 16.704 \\
\hline 2 & 1 & 16.897 \\
\hline 2 & 2 & 14.759 \\
\hline 3 & 1 & 16.600 \\
\hline 3 & 2 & 15.900 \\
\hline
\end{tabular}

La gráfica 7 muestra la representación múltiple del rendimiento obtenido por cada grupo experimental en forma independiente, nivel 1 (level_1) primer aplicación y el nivel 2 (level_2) segunda aplicación. La línea azul corresponde a la curva de rendimiento del grupo 1: en el nivel 1 obtuvo una Media de 19.519 (ver tabla 6) y en el nivel 2 una Media de 16.704 (ver tabla 6) con una diferencia entre Medias de 2.815; la línea roja punteada corresponde a la curva de rendimiento del grupo 2: la Media en el nivel 1 es de 16.879 (ver tabla 6) y en el nivel 2 de 14.759 (ver tabla 6) con una diferencia entre ellas de 2.138; y la línea verde de trazos finos representa la curva de rendimiento del grupo 3: la Media obtenida para el nivel 1 fue de 16.6 (ver tabla 6) y para el nivel 2 de 15.9 (ver tabla 6) existiendo entre ellas una diferencia de 0.700. La diferencia entre Medias de cada grupo experimental, como ya se dijo anteriormente, significan una pérdida de información debida al transcurso del tiempo.

De la gráfica 7 se puede inferir lo siguiente:

- El grupo 1 obtuvo las Medias mayores en las dos aplicaciones del test y por lo tanto hubo mayor retención de la información.

- En los tres grupos experimentales se evidencia una pérdida de información entre la primera y la segunda aplicación del test. El paso del tiempo genera un debilitamiento de las estructuras de memoria que muchos investigadores explican por el fenómeno de interferencia en razón de las experiencias que ocurren en este tiempo.

- Las curvas de rendimiento del grupo 1 y del grupo 2 son similares, sus pendientes son casi idénticas, lo cual significa que hubo una pérdida de información de proporciones semejantes. 
- La curva de rendimiento del grupo 3 mantiene una pendiente menos pronunciada, lo cual indica que la pérdida de información por efecto del paso del tiempo fue mínima comparada con la pérdida de información de los otros dos grupos experimentales; en otras palabras, el texto con animación generó menos pérdida de información con el transcurso del tiempo.

Gráfica 7. Rendimiento de cada grupo experimental.

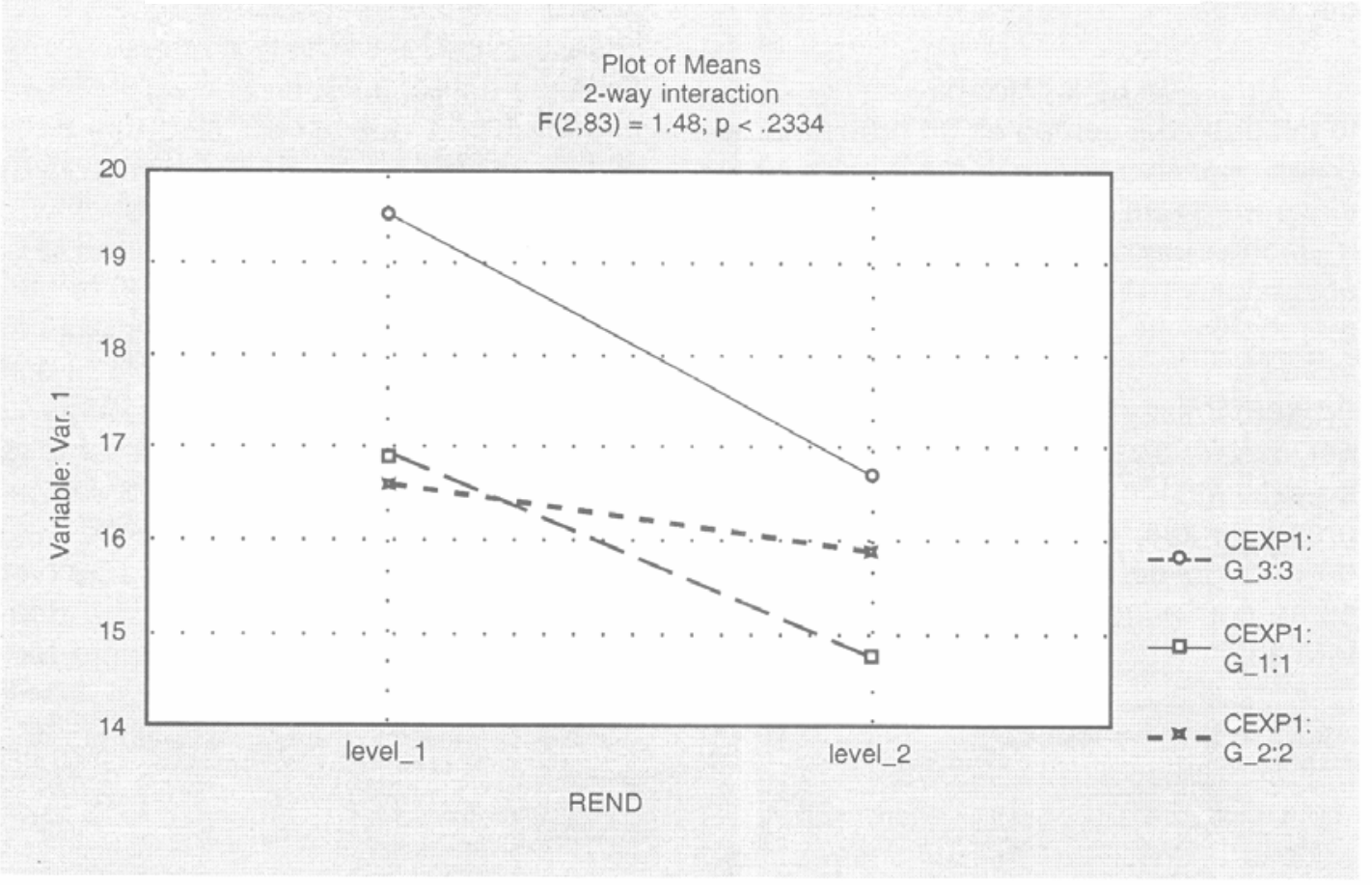

Los datos muestran que el grupo 1 logró una mejor comprensión del texto cuando es complementado con gráficos $\mathrm{y}$, por ende, un mejor rendimiento; pero en contraste, la perdida de la información con el transcurso del tiempo fue mayor. Los que trabajaron con el simulador, aunque las Medias de rendimiento fueron menores, la información se conservó mejor con el transcurso del tiempo, lo cual nos motiva a formular la siguiente pregunta ¿qué pasará con la representación múltiple y comprensión de mensajes si se modifica la condición del tiempo, es decir, amplía el tiempo de lectura? Es posible que la comprensión del texto acompañada de simulación consuma más tiempo, pero que el simulador ayude a organizar mejor la información y en consecuencia a potenciar el almacenamiento en memoria de largo plazo y se facilite la recuperación ulterior de la información. Desde luego esta hipótesis debe ser probada.

Los datos obtenidos muestran que la estrategia de combinar un texto base con gráficos o esquemas explicativos facilita la comprensión de los mensajes, de tal manera que en el mismo tiempo se logran mejores resultados que con el solo texto. Es decir, que la combinación de información visual y textual da soporte a la adquisición de información semántica.

Los resultados sugieren que los diagramas ayudan al proceso cognitivo debido a su especificidad limitando la abstracción (Sternnin \& Oberlander, 1959), también podemos decir que los gráficos conllevan en sí información condensada, fácil de entender y Digitalizado por RED ACADEMICA 
recordar, son un tipo de escritura especial que llevan inmersos modelos representacionales de la solución de problemas, siendo por lo tanto fáciles de interpretar y transformar en una descripción verbal.

Al tratar de comprender un texto sin tener los conocimientos previos del tema, se requiere un cambio en los modelos mentales de los sujetos (Yulin Qin y Herbert Simon, 1995) para procesar y asimilar la nueva información presentada en el material de trabajo, tal es el caso del grupo 2 que necesitó hacer una fuerte simulación mental (Mary Hegarty, 1995) para formarse la imagen del dispositivo o dispositivos descritos dentro del contenido del texto base para organizar y estructurar la nueva información en la memoria a corto y largo plazo, como también para estructurar los conocimientos previos con los nuevos conocimientos y así, lograr una completa comprensión del texto.

La gráfica 8 muestra las frecuencias de respuestas o aciertos para cada una de las preguntas en las dos aplicaciones del test. Sobre el eje $X$ se tabuló el número de casos o número de preguntas, y sobre el eje $Y$ el número de veces que fue acertada cada una de las preguntas. Las barras de color azul corresponden a los aciertos obtenidos en la primera aplicación y las barras de color rojo corresponden a los aciertos obtenidos en la segunda aplicación.

En la gráfica 8 se observa que el caso 40 obtuvo cero (0) aciertos en las dos aplicaciones. El texto de la pregunta 40 era el siguiente: En un motor eléctrico el cálculo del flujo magnético es directamente proporcional al producto de una constante $k$ por el diámetro interno del estator, por la longitud del paquete de chapas y por la inducción magnética, e inversamente proporcional al número de polos. En el campo de abajo, termine usted de escribir la fórmula matemática correspondiente: $\Phi$ . Al parecer, los sujetos tienen dificultad para relacionar fórmulas matemáticas con texto totalmente plano

Gráfica 8. Distribución de frecuencias para los resultados del test.

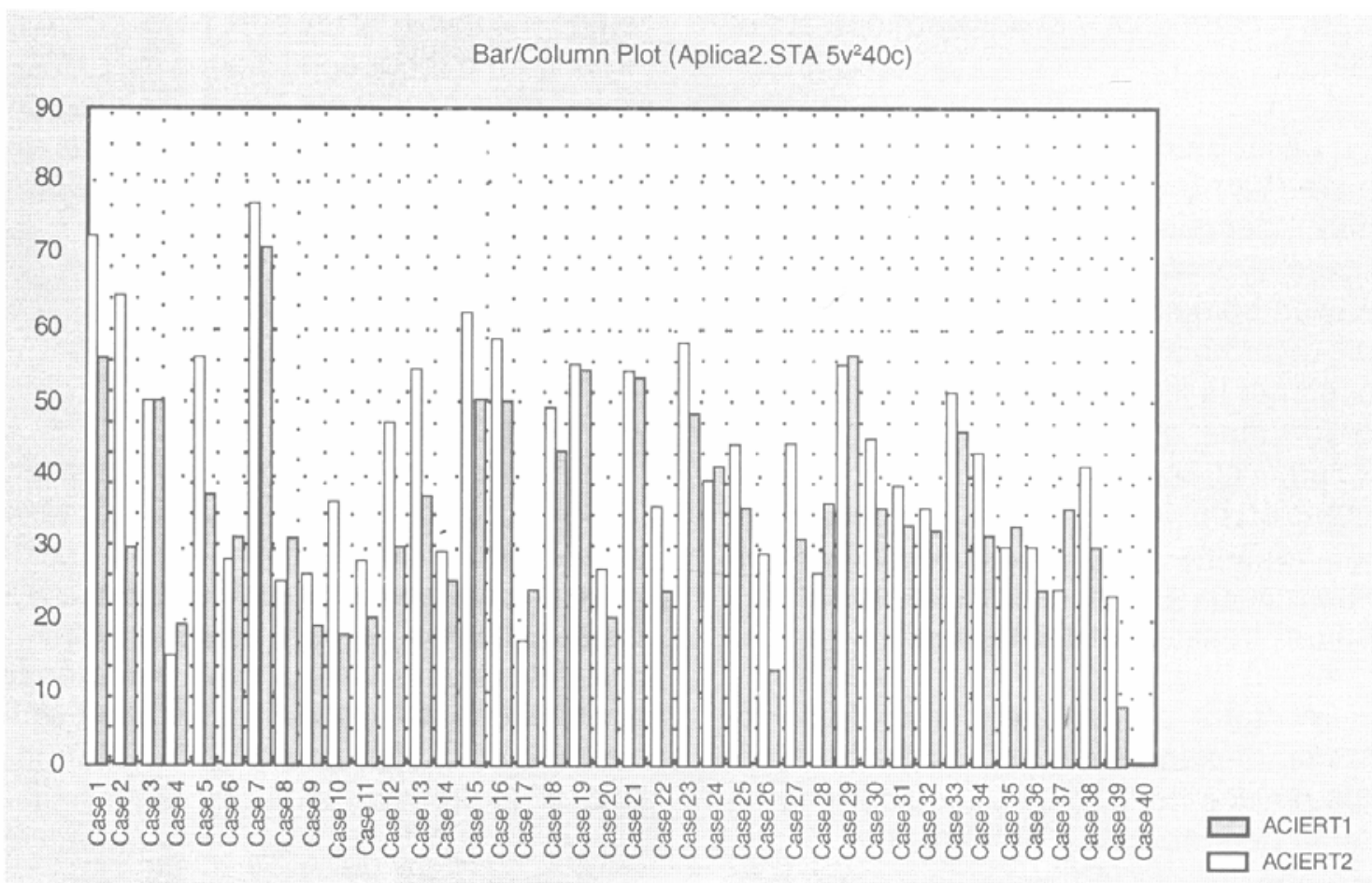




\section{CONCLUSIONES}

Los datos obtenidos en la investigación permiten afirmar que existen diferencias significativas entre los alumnos de que utilizaron para la lectura y comprensión de texto el software gráfico, el software hipertextual y el software con simulación.

La estrategia de combinar un texto base (Van Dijk y Kintsch (1983; Kintsch, 1992)) con sus respectivos gráficos o esquemas explicativos forman una combinación apropiada para la comprensión de un determinado texto, pues, además de contener la literatura propia del tema, los diagramas o esquemas especifican y aclaran el contenido facilitando la comprensión del texto; pero, en contraste con las ventajas descritas, la pérdida de la información en los sujetos es apreciable con el transcurso del tiempo.

Al tratar de comprender un texto totalmente plano sin tener los conocimientos previos del tema, se requiere una total concentración y abstracción, es decir, una disposición a aceptar cambios en los modelos mentales (Yulin Qin y Herbert Simon, 1995) para procesar y asimilar la nueva información, y una fuerte simulación mental (Mary Hegarty, 1995) para representarse la imagen del dispositivo descrito dentro del contenido del texto y relacionar los conocimientos previos con los nuevos en memoria de largo.

En los tres grupos experimentales se manifiesta una pérdida de la información entre la primera y segunda aplicación del test. Los resultados obtenidos demuestran que la estrategia de utilizar un simulador para presentar información técnica se relaciona con una mayor retención relativa en el transcurso del tiempo, en comparación con las otras dos condiciones experimentales. Dado que la animación es portadora de mayor cantidad de información, requiere del lector un mayor trabajo de procesamiento y selección, en contraste con la condición textual o en la condición con diagramas y gráficos donde el escritor presenta sólo la información relevante. Esta interpretación hace prever una mayor demanda de esfuerzo y tiempo por parte del usuario, pero, por otra parte, puede tener condiciones más favorables para establecer asociaciones con información almacenada en memoria de largo plazo. En consecuencia, se puede formular la hipótesis de que, con un mayor tiempo de lectura, los resultados de esta estrategia podrían generar mejores rendimientos relativos tanto en comprensión como en retención.

Finalmente, los resultados sugieren que deducir expresiones matemáticas a partir de un texto totalmente plano constituye una de las mayores dificultades para los estudiantes, lo cual anima a pensar que un sistema que permita relacionar sistemáticamente la representación animada, con la representación diagramática, la expresión textual y la fórmula, puede generar resultados óptimos en la comprensión de información técnica.

\section{BIBLIOGRAFÍA}

Blackwell, Alan F. (1996). "Chasing the Intuition of and Industry: Can Pictures Help Us Think?". In M. Ireland (Ed.): Proceedings of the first Psyohology of Programming Interest Group Postgraduate Student Workshop. Pg. 13-24.

Blackwell, Alan F. (1997). "Diagrams about Thoughts about Thoughts about Diagrams". In M. Anderson, (Ed.) Reasoning with Diagrammatic Representations II: Papers from the 
AAAl 1997 Pall Symposium. Technical Report FS-97-02. Menlo Park, California: AAAI Press. Pg. 77-84.

Finke, R. A.; Pinker, S. y Farah, M. J. (1989). Reinterpreting visual patterns in mental imagely. Cognitive Science 13:51-78.

Glenberg, A. M.; Langston, W. E. (1992). Comprehension of illustrated text: Pictures help to build mental models. Journal of Memory and Language 31:129-151.

Goodman, N. (1969). Languages of art: An approach to a theory of symbols. London: Oxford University Press.

Goel, Vinod y Pirolli, Peter (1992). "The Structure of Design Problems Space". En: Cognitive Science. No. 6, Stanford, Pp. 395-429.

Green T.R.G. y Petre, M. (1996). Usability analysis of visual programming en vironments: a 'cognitive dimensions' approach. Journal of Visual Languages and Computing 7, Pg. 131-174.

Hegarty, M. (1992). Mental animation: Inferring motion from static displays of mechanical systems. Journal of Experimental Psychology: Learning, Memory and Oognition 18, Pg. 1084-1102.

Hegarty, Mary (1995). "Mental Animation. University of California, Santa Barbara". In: Glasgow J., Narayaran N. H. And Chandrasekaran, B. (Edit): Diagrammatic Reasoning. Cognitive and computational perspectives. Cambridge, MA: The MIT Press.

Huttenlocher, J. (1968). "Constructing spatial images: a strategy in reasoning'. In: Psychological Review 75. Pg. 550-560.

Ittelson, W. H. (1996). Visual perception of markings. Psychonomic Bulletin \& Review 3. Pg.171-187.

Kearins, J. M. (1981). "Visual spatial memory in Australian Aboriginal children of desert regions". En: Cognitive Psychology 13. Pg. 434-460.

Kintsch, Walter (1994). "Text Comprehension, Memory, and Learning". In: American Psychologist Association. Vol. 49, No. 4. Pg. 294-303.

Larkin, J. H. y Simon, H. A. (1987). Why a diagram is (sometimes) worth ten thousand words. Cognitive Science 11. Pg. 65-99.

Linde, C. y Labov, W. (1975). Spatialstructures as a site for the study of language and thought. Language, 51. Pg. 924-939.

Mcbride, Allan. "Teaching Research Methods Using Appropriate Technology. Grambling State University. En: CD-ROM, Base de datos "Social Science”, Hemeroteca Nacional. Santafé de Bogotá.

Narayanan, N. Han (1995). "Hypothesizing Behaviors from Device Diagrams. Georgia Institute of Technology. In: Glasgow J., Narayaran N. H. And Chandrasekaran, B. (Edit): 
Diagrammatic Reasoning. Cognitive and computational perspectives. Cambridg, MA: The MIT Press.

Nilsson, N. J. (1971). Problem solving methods in artificial intelligence. New York: MacGraw Hill Edit.

Otero, J. (1990). "Variables cognitivas y metacognitivas en la comprensión de textos científicos: el papel de los esquemas y el control de la propia comprensión". En: Revista Enseñanza de las Ciencias, Vol. 8., No 1, marzo de 1990.

Qin, Yulin y Simon, Herbert (1995). "Imagery Mental Models. Carnegie Mellon University. In: Glasgow J., Narayaran N. H. And Chandrasekaran, B. (Edit): Diagrammatic Reasoning. Cognitive and computational perspectives. Cambridg, MA: The MIT Press.

Rosenberg, Robert (1970). Reparación de motores eléctricos. Barcelona: Ed. Gustavo Gili, S.A.

Schooler, J. W.; Ohlsson, 5. y Brooks, K. (1993). Thoughts beyond words: When language overshadows insight. Journal of Experimental Psychology, 122. Pg.166-183.

Schwartz, D. L. (1995). Reasoning about the referent of a picture versus reasoning about the picture as the referent: An effect of visual realism. Memory and Cognition, 23 . Pg. 709-722.

Siemens (1998). Motores eléctricos de inducción. Bogotá: Folleto técnico.

Singer, Francisco L. (1976). Tratado de bobinados. Buenos Aires: Neotecnica Edit.

Smyth, M. M. y Scholey, K. A. (1996). The relationship between articulation time and memory performance in verbal and visuospatial tasks. British Journal of Psychology, 87. Pg. 179-191.

Suwa, Masaki y Motoda, Hiroshi (1995). "Hypothesizing Behaviors from Device Diagrams. Hitachi Advanced Research Laboratory". In: Glasgow J., Narayaran N. H. And Chandrasekaran, B. (Edit): Diagrammatic Reasoning. Cognitive and computational perspectives. Cambridg, MA: The MIT Press. 